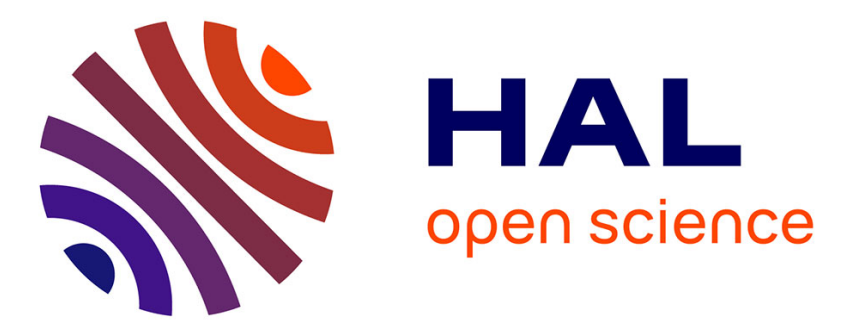

\title{
Treewidth of planar graphs: connections with duality
}

Vincent Bouchitté, Frédéric. Mazoit, Ioan Todinca

\section{To cite this version:}

Vincent Bouchitté, Frédéric. Mazoit, Ioan Todinca. Treewidth of planar graphs: connections with duality. Euroconference on Combinatorics, Graph Theory and Applications, Sep 2001, Barcelona, France. pp.34-38, 10.1016/S1571-0653(04)00353-1 . hal-00351167

\section{HAL Id: hal-00351167 https://hal.science/hal-00351167}

Submitted on 8 Jan 2009

HAL is a multi-disciplinary open access archive for the deposit and dissemination of scientific research documents, whether they are published or not. The documents may come from teaching and research institutions in France or abroad, or from public or private research centers.
L'archive ouverte pluridisciplinaire HAL, est destinée au dépôt et à la diffusion de documents scientifiques de niveau recherche, publiés ou non, émanant des établissements d'enseignement et de recherche français ou étrangers, des laboratoires publics ou privés. 


\title{
Treewidth of planar graphs: connection with duality
}

\author{
Vincent Bouchitté ${ }^{1}$, Frédéric Mazoit $^{1}$, and Ioan Todinca ${ }^{2}$ \\ 1 LIP-École Normale Supérieure de Lyon, 46 Allée d'Italie, 69364 Lyon Cedex 07, \\ France, \{Vincent.Bouchitte, Frederic.Mazoit\}@ens-lyon.fr \\ 2 LIFO - Université d'Orléans, BP 6759, 45067 Orléans Cedex 2, France \\ Ioan.Todinca@lifo.univ-orleans.fr
}

\section{Preliminaries}

A graph is said to be chordal if each cycle with at least four vertices has a chord, that is an edge between two non-consecutive vertices of the cycle. Given an arbitrary graph $G=(V, E)$, a triangulation of $G$ is a chordal graph $H(=V, F)$ such that $E \subseteq F$. We say that $H$ is a minimal triangulation of $G$ if no proper subgraph of $H$ is a triangulation of $G$. The treewidth $\operatorname{tw}(H)$ of a chordal graph is its maximum cliquesize minus one. The tree-width of an arbitrary graph $G$ is the minimum, over all triangulations $H$ of $G$, of $\operatorname{tw}(H)$. When computing the treewidth of $G$, we can clearly restrict to minimal triangulations. Treewidth was introduced by Robertson and Seymour in connection with graph minors [5], but it has wide algorithmic applications since many NP-hard problems become polynomial when restricted to graphs of bounded treewidth.

Robertson and Seymour conjectures in [5] that the treewidth of a planar graph $G$ and its dual $G^{*}$ differ by at most one. This conjecture was recently proved by Lapoire [3], who gives a more general result, on hypergraphs of bounded genus. Nevertheless, the proof of Lapoire is rather long and technical. Here, we show that any minimal triangulation $H$ of a planar graph $G$ wan be easily transformed into a triangulation $H^{*}$ of $G^{*}$. such that $\operatorname{tw}\left(H^{*}\right) \leq \operatorname{tw}(H)+1$.

Theminimal separators play a crucial role in the characterisation of the minimal triangulations of a graph. A subset $S \subseteq V$ separates two non-adjacent vertices $a, b \in V$ is $a$ and $b$ are in different connected components of $G \backslash S$. $S$ is a minimal $a, b$-separator if it separates $a$ and $b$ and no proper subset of $S$ separates $a$ and $b$. We say that $S$ is a minimal separator of $G$ if there are two vertices $a$ and $b$ such that $S$ is a minimal $a, b$-separator. Notice that a minimal separator can be strictly included into another. We denote by $\Delta_{G}$ the set of all minimal separators of $G$. Two minimal separators $S$ and $T$ cross if $T$ intersects at least two components of $G \backslash S$. Otherwise, $S$ and $T$ are parallel. Both relations are symmetric.

Let $S \in \Delta_{G}$ be a minimal separator. We denote by $G_{S}$ the graph obtained from $G$ by completing $S$, i.e. by adding an edge between every pair of non-adjacent vertices of $S$. If $\Gamma \subseteq \Delta_{G}$ is a set of separators of $G, G_{\Gamma}$ is the graph obtained by completing all the separators of $\Gamma$. The result of [2], concluded in [4], establish a 
strong relation between the minimal triangulations of a graph and its minimal separators.

Theorem 1. $H$ is a minimal triangulation of $G$ if and only if there is a maximal set of pairwise parallel separators $\Gamma \subseteq \Delta_{G}$ such that $H=G_{\Gamma}$.

Since it is easy to extend our results to simply connected or disconnected graphs, we will restrict to 2-connected graphs.

\section{Minimal separators in planar graphs}

Consider a 2-connected planar graph $G=(V, E)$. We fix an embedding of $G$ in the plane $\mathbb{R}^{2}$. Let $F$ be the set of faces of this embedding. Let $F$ be the set of faces of this embedding. The intermediate graph $G_{I}$ has vertex set $V \cup F$. We place an edge in $G_{I}$ between an original vertex $v \in V$ and a face $f \in F$ whenever the corresponding vertex and face are incident in $G$. Notice that $\left(G^{*}\right)_{I}=G_{I}$.

Let $\nu$ be a cycle of $G_{I}$ (by "cycle" we will always mean a cycle which does not get through a same vertex twice). The drawing of $\nu$ forms a Jordan curve in the plane $\mathbb{R}^{2}$, denoted $\tilde{\nu}$. It is easy to see that if $\tilde{\nu}$ separates two original vertices $x$ and $y$ in the plane (i.e. $x$ and $y$ are in different regions of $\mathbb{R}^{2} \backslash \nu$ ), then $v \cap V$ separates $x$ and $y$ in $G$. Therefore, the original vertices of $\nu$ form a separator in $G$. Conversely, to each minimal separator $S$ of $G$, we can associate a cycle $\nu$ of $G_{I}($ see $[1])$.

Proposition 1. Let $S$ be a minimal separator of the planar graph $G$. Consider two connected components $C$ and $D$ of $G \backslash S$. There is a cycle $\nu_{S}$ of $G_{I}$ such that $\tilde{\nu}$ separates $C$ and $D$ in the plane.

This cycle is usually not unique. In the case of 3-connected planar graphs, notice that if $S$ is a minimal separator, then $G \backslash S$ has exactly two connected components $C$ and $D$. For each couple of original vertices $x$ and $y$ incident to a same face, fix a unique face $f(x, y)$ containing both $x$ and $y$. We say that a cycle $\nu$ of $G_{I}$ is well-formed if, for any two consecutive original vertices $x, y \in \nu$, the face-vertex between them if $f(x, y)$. If $G$ is a 3-connected planar graph, for any minimal separator $S$, there is a unique well-formed cycle of $G_{I}$ separating $C$ and $D$ in the plane.

In what follows, $\boldsymbol{G}$ denotes a 3-connected planar graph. However, our main results can be easily extended to arbitrary planar graphs.

We say that two Jordan curves $\tilde{\nu}_{1}$ an $\tilde{\nu}_{2}$ cross if $\tilde{\nu}_{1}$ intersects the two regions defined by $\tilde{\nu}_{1}$. Otherwise, they are parallel. Two cycles $\nu_{1}$ and $\nu_{2}$ of $G_{I}$ cross if and only if $\tilde{\nu}_{1}$ and $\tilde{\nu}_{2}$ cross. Notice that the parallel and crossing relations between curves and cycles are symmetric.

Proposition 2. Two minimal separators $S$ and $T$ of $G$ are parallel if and only if the corresponding cycles $\nu_{S}$ and $\nu_{T}$ of $G_{I}$ are parallel. 
Let $\tilde{\nu}$ be a Jordan curve in the plane. Let $R$ be one of the regions of $\mathbb{R}^{2} \backslash \tilde{\nu}$. We say that $(\tilde{\nu}, R)=\tilde{\nu} \cup R$ is a one-block region of the plane, bordered by $\tilde{\nu}$. Let $\tilde{\mathcal{C}}$ be a set of curves such that for each $\tilde{\nu} \in \tilde{\mathcal{C}}$, there is a one-block region $(\tilde{\nu}, R(\tilde{\nu}))$ containing all the curves of $\tilde{\mathcal{C}}$. We define the region between the elements of $\mathcal{C}$ as $R B(\mathcal{C})=\cap_{\tilde{\nu} \in \tilde{\mathcal{C}}}(\tilde{\nu}, R(\tilde{\nu}))$. A subset $B r \subseteq \mathbb{R}^{2}$ of the plane is a block region if $B R$ is a one-block region $(\tilde{\nu}, R)$ or $B R$ is the region between some set of curves $\tilde{\mathcal{C}}$.

\section{Minimal triangulations of $G$ and $G^{*}$}

Let $G$ be a 3-connected planar graph and let $H$ be a minimal triangulation of $G$. According to Theorem 1, there is a maximal set of pairwise parallel separators $\Gamma \subseteq \Delta_{G}$ such that $H=G_{\Gamma}$. Let $\mathcal{C}(\Gamma)=\left\{\nu_{S} \mid S \in \Gamma\right\}$ be the cycles of $G_{I}$ associated to the minimal separators of $\Gamma$ and let $\tilde{\mathcal{C}}(\Gamma)=\left\{\tilde{\nu}_{S} \mid S \in \Gamma\right\}$ be the curves associated to these cycles. According to Proposition 2, the cycles of $\mathcal{C}(\Gamma)$ are pairwise parallel. Thus, the curves of $\tilde{\mathcal{C}}(\Gamma)$ split the plane into block regions. Consider the set of all the block regions bordered by some elements of $\tilde{\mathcal{C}}$. We show that any maximal clique $\Omega$ of $H$ corresponds to the original vertices contained in a minimal block regions defined by $\tilde{\mathcal{C}}(\Gamma)$.

Theorem 2. Let $G$ be a 3-connected planar graph and let $H=G_{\Gamma}$ be a minimal triangulation of $G . \Omega \subseteq V$ is a maximal clique of $H$ if and only if there is a minimal block region $B R$ defined by $\tilde{\mathcal{C}}(\Gamma)$. such that $\Omega=B R \cap V$.

Let now $\mathcal{C}$ be an arbitrary set of pairwise parallel cycles of $G_{I}$. This family $\tilde{\mathcal{C}}$ of curves associated to these cycles splits the plane into block regions. Let $G^{*}$ be the dual of $G$. The graph $H^{*}(\mathcal{C})=\left(F, E_{H}\right)$ has vertex set $F$. We place an edge between two face-vertices $f$ and $f^{\prime}$ of $H$ if and only if $f$ and $f^{\prime}$ are in a same minimal block region defined by $\tilde{\mathcal{C}}$. Equivalently, $f$ and $f^{\prime}$ are non-adjacent in $H^{*}(\mathcal{C})$ if and only if there is a $\tilde{\nu} \in \tilde{\mathcal{C}}$ separating $f$ and $f^{\prime}$ in the plane.

Theorem 3. $H^{*}(\mathcal{C})$ is a triangulation of $G^{*}$. Moreover, any clique $\Omega^{*}$ of $H^{*}$ is contained in some minimal block region $B R$ defined by $\tilde{\mathcal{C}}$.

Let $H=G_{\Gamma}$ be a minimal triangulation of $G$. Consider the cycles $\mathcal{G}(\Gamma)$ associated to the minimal separators of $\Gamma$ and the corresponding curves $\tilde{\mathcal{C}}(\Gamma)$. We could try to considerate the triangulation $H^{*}(\mathcal{C}(\Gamma))$ of $G^{*}$, but unfortunately it does not satisfy $\operatorname{tw}\left(H^{*}\right) \leq \operatorname{tw}(H)+1$.

Thus, we consider a maximal set of pairwise parallel cycles $\mathcal{C}^{\prime}$ of $G_{I}$ such that $\mathcal{C}(\Gamma) \subseteq \mathcal{C}^{\prime}$. Clearly, each minimal block region defined by $\mathcal{C}^{\prime}$ is contained in a minimal block region defined by $\tilde{\mathcal{C}}(\Gamma)$.

Theorem 4. Let $\mathcal{C}^{\prime}$ be a maximal set of pairwise parallel cycles of $G_{I}$. Let $B R$ be a minimal block region of $\mathcal{C}^{\prime}$. Then $B r \cap G_{I}$ is either formed by a cycle $\tilde{\nu}$ and a path $\tilde{\mu}$ joining two vertices of $\tilde{\nu}$ or $B R$ is a one-block region $(\tilde{\nu}, R)$ and $B R \cap G_{I}=\nu$ where $\nu$ is the cycle of $G_{I}$ associated to $\tilde{\nu}$. In particular, $\left|B R \cap V^{*}\right| \leq|B R \cap V|+1$. 
According to theorem 3, each maximal clique $\Omega^{*}$ of $H^{*}$ is contained in some minimal block region $B R$, and by the previous theorem it has at most one more vertex than $\Omega=B R \cap V$. By theorem $2, \Omega$ is a clique of $H$. Hence, $\left|\Omega^{*}\right| \leq|\Omega|+1$ and thus $\operatorname{tw}\left(H^{*}\right) \leq \operatorname{tw}(H)+1$. By considering an optimaltriangulation $H$ of $G$, we obtain a triangulation $H^{*}$ of $G^{*}$ of width at most $\operatorname{tw}(G)+1$. We conclude that $\operatorname{tw}\left(G^{*}\right) \leq \operatorname{tw}(G)+1$.

So we can state:

Theorem 5 (Main theorem). Let $G=(V, E)$ be a planar graph.

$$
\left|\operatorname{tw}(G)-\operatorname{tw}\left(G^{*}\right)\right| \leq 1 \text {. }
$$

\section{References}

1. D. Eppstein. Subgraph isomorphism in planar graphs and related problems. Journal of Graph Algorithms and Applications, 3(3):1-27, 1999.

2. T. Kloks, D. Kratsch, and H. Müller. Approximating the Bandwidth for Asteroidal Triple-Free Graphs. Journal of Algorithms, 32:41-57, 1999.

3. D. Lapoire. Treewidth and duality for planar hypergraphs. Manuscript: http://www . labri.fr/perso/lapoire/papers/dual_planar_treewidth.ps, 1996.

4. A. Parra and P. Scheffler. Characterizations and algorithmic applications of chordal graph embeddings. Discrete Applied Mathematics, 79(1-3):171-188, 1997.

5. N. Robertson and P. D. Seymour. Graph Minors. III. Planar Tree-Width. Journal of Combinatorial Theory Series B, 36(1):49-64, 1984. 